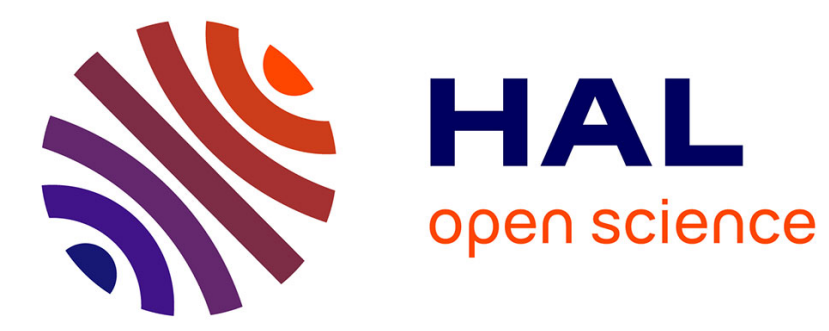

\title{
Basic concepts of the approach by continuum mechanics
}

P. Germain

\section{To cite this version:}

P. Germain. Basic concepts of the approach by continuum mechanics. Revue de Physique Appliquée, 1988, 23 (4), pp.319-323. 10.1051/rphysap:01988002304031900 . jpa-00245776

\section{HAL Id: jpa-00245776 https://hal.science/jpa-00245776}

Submitted on 1 Jan 1988

HAL is a multi-disciplinary open access archive for the deposit and dissemination of scientific research documents, whether they are published or not. The documents may come from teaching and research institutions in France or abroad, or from public or private research centers.
L'archive ouverte pluridisciplinaire HAL, est destinée au dépôt et à la diffusion de documents scientifiques de niveau recherche, publiés ou non, émanant des établissements d'enseignement et de recherche français ou étrangers, des laboratoires publics ou privés. 


\title{
Basic concepts of the approach by continuum mechanics
}

\author{
P. Germain
}

Laboratoire de Mécanique Théorique, Université Pierre et Marie Curie, 75005 Paris, France

(Reçu le 26 mai 1987, accepté le 22 janvier 1988)

\begin{abstract}
RESUME - On m'a demandé pour initier cette réunion de scientifiques de diverses disciplines, destinée à comprendre les mécanismes de la plasticité, de rappeler les points de départ, les méthodes et les objectifs de l'approche mécanique.

ABSTRACT - I have been asked, at the beginning of this meeting of scientists of various disciplines which is devoted to the understanding and to the mastering of the mechanisms of plasticity, to recall the starting points, the methods and objectives of the approach from mechanics.
\end{abstract}

I. The Laws of the interactions

I.1. Kinematical description

Mechanics wants to give a continuation of the geometrical representation in order to explain and to predict the motion -or the equilibrium- of bodies and structures subjected to various physical or chemical interactions. Anv body $B$ may be viewed as a set of element $M$, usually assumed to be a continuous set, provided the number of these elements is great and their mutual distances small. Mechanics, namely, always tries to start with the most simple representation in order to go as far as possible into the predictions. At each time $t$, one is looking for the displacement field $\underset{\sim}{X}(t)$ of the various elements of $B$. Usually $M$ is a point and $\underset{\sim}{X}$ the field of the displacement vector $\vec{X}(M, t)$ from a reference configuration (Lagrange). But sometimes the element $M$ has to be considered -say- as the schematization of a rigid body (micropolar continuum). The field $\underset{\sim}{x}$ is then the field $\vec{x}(t)$ generated not only by a displacement vector but also by an orthogonal tensor which gives the orientation of the rigid bodies. As it is well known, one may recover $X(t)$ from the "velocity" field $\underset{\sim}{U}$ (Euler) of the $\vec{U}(M, t)$ which defines the "velocity" of the element $M$.

I.2. Mechanical interactions

The most natural way to define mechanical actions on a body $B$-the decisive and completely new concept on which classical mechanics is based- is to say how this body reacts under this action for various kind of instantaneous small motions -one raises a little bit a suitcase if one whishes to evaluate its weight, one moves a little bit the belt of the ventilator of a car in order to see if it is conveniently stressec. In mathematical words, if $\hat{U}$ is a (virtual) velocity field, defined on $B$ at time $t$, as an element of a vector space of virtual motions $V$, the mechanical actions of a system $S$ on $B$ may be defined, for this class of v.m. $V$, by its virtual power -a real number : $\hat{P}=L(\underset{\sim}{\tilde{U}})$

where $L$ is a linear and continuous function on $V$.

As a consequence, if $B$ is a finite set of points these actions are defined by a vector field on $B$, i.e. by forces. One recovers the Newton's description, usually adopted by physicists. Vector fields give namely a quite convenient representation of the mechanical actions of a system $S$ on a body $B$; but mutual mechanical interactions inside $B$-stressesare not represented by vector fields, but by a scalar field in a fluid in equilibrium or a field of symmetric second order tensors in classical continuum mechanics.

The definition (1) provides in any case the best way to introduce the definition of stresses. It 
rests on the objectivity statement which says that mutual mechanical interactions must be invariant by any change of referential frame. Then, in (1), the ingredients coming from $\underset{\sim}{U}$ must be objective quantities $\hat{D}$, say

$$
\hat{\sim}=\Delta(\hat{\sim})
$$

In classical continuum mechanics $\vec{D}=(\overrightarrow{\nabla U})_{\mathrm{s}}$, the symetric part of the virtual velocity gradient. Then the stress field $\underset{\sim}{\sigma}$ appears as the dual quantity of $\hat{D}$. More precisely (1) may be written

$$
-\hat{P}_{(i)}=\left(\hat{D}_{\sim}, \sigma\right)
$$

if $\hat{P}_{(i)}$, is the virtual power of the mutual interactions. One checks immediately that, in classical contiunuum mechanics, $\sigma$ is the field of stress tensor $\vec{\sigma}$. In this case, $-\hat{p}_{(i)}$ is namely the volume ingral of the trace of the product of the matrices $\underline{\sigma}$ and $\underline{\bar{D}}$ :

$$
\hat{\varepsilon}_{(i)}=\operatorname{tr}(\underline{\sigma} \underline{\underline{D}})
$$

The fundamental statement of statics say that in any ralilean frame, the nower of the external force $F$ (exerted on $B$ by systems exterior to $B$ ) - say $\hat{P}_{(e)}^{2}$ and of internal forces - say $\hat{P}_{(i)}$ - is always null. Accordingly :

$$
(\underset{\sim}{\hat{U}}, \underset{\sim}{F})=(\stackrel{\hat{D}}{\sim}, \underset{\sim}{\sigma})
$$

whatever be $\hat{U}$ in $\%$. Then, if $\Delta^{*}$ is the adjoint operator of $\Delta$ in (2),

$$
\underset{\sim}{F}=\Delta^{*} \underset{\sim}{(\sigma)}
$$

represents the equations of equilibrium. The main results, whose derivations has been outlined above, may be condensed in the followina sketch :

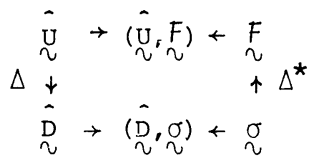

I.3. Thermomechanical interactions and other interactions

Usually other interactions than the mechanical ones have to be taken into account in order to study the motion -or the equilibrium- of a body. The most commun ones are the thermomechanical interactions. The basic statement here is the conservation of energv, a natural extension of the first law of thermostatics, which says that for any closed body, the time derivation of the sum of the internal energv and of the kinetic energy is equal to the power of exterior forces $P_{e}$ and of the rate of heat received by the bodv. Isina the theorem of mechanics on kinetic ener- gy, one can get rid of this energy as well as of $P_{(e)}$, and obtain locally :

$$
\dot{e}=\varepsilon_{(i)}+\varepsilon_{(t h)}
$$

where $e$ is the specific internal enerqy (per unit mass), $\dot{e}$, the rate of $e, \tilde{\varepsilon}_{(i)}$, the power due to internal stresses p.u.m., $\tilde{\varepsilon}_{\text {th }}$ the heat rate receveid by the element $M$ and o.u.m., in particular due to thermal conduction onlvif, as usually, radiative heat is neglected. In classical mechanics (see (4)), if $\rho$ is the volumic mass (p.u.v.) :

$$
\tilde{\varepsilon}_{(i)}=\varepsilon_{(i)}=(\vec{\sigma}, \vec{D})=\operatorname{tr}(\underline{\sigma} \underline{D}), \tilde{\varepsilon}_{(\operatorname{thr})}^{\tilde{d i v q}(9)}
$$

if $\vec{q}$ is the heat flux vector.

Various physical and chemical interactions, beyond thermomechanical ones, may have to be taken into account. Electromagnetic phenomena occur in magneto-fluid dynamics or in piezoelectricty ; optomechanics is a new field with some interesting applications, multiphasic flows, flows of dissociated fluids or ionized fluids, motions of reactive media are typical examples of cases which require the introduction of other convenient physical or chemical laws in order to have, with the basic equations of motion or of equilibrium, the necessary basis for studying the motions or equilibrium of the bodies which are subjected to such phenomena.

The choice of the basic laws which are assumed to give a sufficentlv accurate descriotion of the phenomena involved -in particular the choice of the representation of stresses and forces as qiven in (6), i.e. the choice of. $\underset{\sim}{U}$ and of $\Delta$-defines the basic equations (and consequently the refinement) of the theory which will be applied for the applications one has in mind. They are sometimes called the universal equations because, to a large extent, they are independant of the properties of the materials involved.

II. The thermodynamical frame of constitutive equations.

It is quite clear that the lavs of the basic laws of the various interactions to which the body is subjected must be comoleted by the knowledae of the properties of the material of $\mathrm{B}$ itself. In a mechanical representation, this knowledre is schematized in the constitutive laws. To discover the deneral properties of these laws, the special ones they have to fulfil for each class of materials and the special ingredients that have to be taken into account for the material of a bodv deformed under a 
particular trme of loading, has been one the main task of continuum mechanics these last forty years and the field of some of its more spectacular achievments.

These laws qive the various quantities which rule the transfers inside the body B - momentum transfer (stress tensor); heat transfer (heat flux vector) etc...

It has been recoqnized at the early staces of the study of constituve laws that they must be ohjective (orinciole of material indifference) and that at time $t$ they must depend only of the past historv un to time $t$ (orincinle of causalitỵ). Twentv vears aqo it becomes clear, for most of the peonle working in this field, that classical (or phenomenolocical) thermodynamics is the discioline which may provide a good conceptual frame for the formulation of constitutive equations. One will follow in the present review the so-called "local accompanying state" (1.a.s.) approach.

Let us note, to begin with, that the power in (8) can always be written

$$
\tilde{\varepsilon}_{(i)}=(\tilde{c}, \dot{\delta})
$$

where $\delta$ is the material derivative of a quantity $\delta$ which represents the deformation and $\tilde{c}$ the stress representation conjugate to $\dot{\delta}$; the $(.$, .) is as usual the canonical bilinear form. In the classical small perturbation theorv (s.p.t.), $\delta$ is simply $\underline{\varepsilon}$, the s.p.t. deformation matrix and $\tilde{\rho c}$ is the stress matrix $\sigma$; for finite deformation, one may take for $\delta$ the Green - Lagrange deformation tensor and then $\widetilde{C}$ is proportionnal to the symmetric Piola-Kirchhoff stress tensor.

II.1. Elastic or perfect materials one assumes that to any particule, one may associate a thermodynamical homogeneous system of unit mass whose states may be described by the variable $\delta$ and s - a specific entropy. The reversible evolutions of this system are assumed to be ruled by the thermodynamical potentiel and the first law of thermodynamics for this system gives exactly the same equation as (8), then

$$
\tilde{\varepsilon}_{(i)}=(\tilde{c}, \dot{\delta}), \quad \tilde{\varepsilon}_{(t h)}=T \dot{s}
$$

consequently

$$
\tilde{c}=\frac{\partial e}{\partial \delta}(\delta, s) \quad, \quad T=\frac{\partial e}{\partial s}(\delta, s)
$$

This assumption gives the possibility to define for any particule of $B$ the specific entropy $s$, the absolute temperature $T$ and to obtain the equations of state (12). Of course, one mav choose another thermodynamical notentiel for describina the 1.a.s., for instance the specific freee energy $\psi(\delta, T)$. Then (12) is replaced by

$$
\tilde{c}=\frac{\partial \psi}{\partial \delta}(\delta, T) \quad, s=-\frac{\partial \psi}{\partial T}(\delta, T), e=\psi+T s(13)
$$

It is easily checked that for an isothermal evolution ( $T$ constant), (13)1 qives the full constitutive equations and for an adiabatic evolution, as (9) and (11) show that s remains constant, equation (12) gives the full constitutive equations.

Note that for a perfect fluid $\delta$ may be taken simply as the specific volume $\tau, \widetilde{C}$ is then $-p$, with p the pressure.

\section{II.2. Anelastic materials}

One restricts the analysis to the following situation. To any particule of $B$, one assumes that one may associate a thermodynamical system of unit mass whose states may be described by the variables $\delta$ and $s$, as above, but also by a set of internal variables $\alpha$. The reversible evolution of this system are ruled by a potential e $(\delta, s, \alpha)$ for which the first law is written

$$
\begin{aligned}
& \left.\dot{\mathrm{e}}=\tilde{\omega}_{(i)}+\mathrm{T} \dot{\mathrm{s}}, \tilde{\omega}_{(i)}=\tilde{(c}_{\mathrm{c}}, \dot{\delta}\right)-\tilde{(\tilde{A}}_{,}, \dot{\alpha}_{)}=\mathrm{T} \varepsilon_{(i)}- \\
& (\tilde{\mathrm{A}}, \dot{\alpha})
\end{aligned}
$$

where $\dot{e}$ is exactly the same as in (8). As a result, one may write the equations of state (E.S.)

$\widetilde{c}=\frac{\partial e}{\partial \delta}(\delta, s, \alpha), T=\frac{\partial e}{\partial s}(\delta, s, \alpha),-\widetilde{A}=\frac{\partial e}{\partial \alpha}(\delta, s, \alpha)(15)$ in a form which generalizes (12) or in another one, with the specific freee energy, in a form which generalizes (13). Moreover

$$
(\tilde{A}, \dot{\alpha})=\tilde{\varepsilon}_{(i)}-\tilde{\omega}_{(i)}=\mathrm{T} \dot{s}-\tilde{\varepsilon}_{(t h)}
$$

It then appears that the real evolutions of the particle cannot be considered to be reversible even if the evolutions of the (fictitious) 1.a.s. are. Moreover the equations of state (15) don't provide the full consitutive equations. They must be completed by some complementary constitutive equations (C.C.E.). The fundamental inequality of thermodynamics applied to the 1.a.s. as given by ClausiusDuhem inequality (in order to eliminate every influence of external effects) which mav he written with $\tilde{\varepsilon}_{\text {th }}$ aiven by (9) and with $\tilde{\rho} \tilde{A}=A$ :

$$
(A, \dot{\alpha})-\vec{q} \cdot \vec{\xi} \geqslant 0, \vec{\xi}=\nabla(\log T)
$$

Here $(A, \dot{\alpha})$ is the intrinsic dissipation D.u.v. which is due to the irreversible properties of internal power of stresses $\varepsilon_{(i)}-\omega_{(i)} ;-\vec{q} \cdot \vec{\xi}$ is the 
thermal dissipation p.u.v., due to the heat transfer exchanges in a nonhomogeneous temperature field. The C.C.E., one is lookina for, must generate thermodynamical admissible processes, that means processes for which (17) is always verified.

one usually write a Fourier law for heat conduction :

$$
\overrightarrow{\mathrm{q}}=-\mathrm{K}(\mathrm{T}) \cdot \vec{\xi}
$$

where $\vec{K}$ is a symmetric second order tensor positive definite, a law which rules the thermal dissipation. For the intrinsic one, one may assume a ceneral evolution equation like :

$$
\dot{\alpha}=f(\lambda ; \alpha, \pi, \delta)
$$

But very often, it is possible to express $\dot{\alpha}$ as a gradient (or a subgradient) of a pseudopotential of dissipation $\left(?^{*}(A)\right.$ - which may also depend of some thermodynamical variable or $A$ as a gradient of a pseudo-potential $\because(\dot{\alpha})$

$$
\dot{\alpha}=\partial_{\mathrm{A}} \varphi^{*}(\mathrm{~A}) \quad, \quad \underline{A}=\partial_{\dot{\alpha}} \varphi(\dot{\alpha})
$$

with $\varphi$ and $\varphi)^{*}$ two dual convex functions with $\varphi^{*}(0)=\partial_{A}\left(^{*}(0)=0\right.$. One then says that the dissiontion is normal or that the materials is standard. All processes for such a standard material whose C.C.E. are given by (18) and (20) are thermodynamically admissible.

Let us emphasize that the above analysis gives an interesting frame for writing constitutive equations. If, for instance, the standard material model may be adopted, one has to define the internal variables, to find the free energy $\psi$, or equivalenty the equations of state, to find the pseudopotential $\varphi^{*}(A)$. If such a orogram may be fulfilled, one may hope to be able to formulate well-posed mathematical problems in order to find the behavior of $B$, to study their solutions together with their evolution and their stability for various data. One will be able then to test the validity of the model, i.e. its capacity to explain various experimentally observed phenomena and to give quantitative evaluations which may be compared to measurements.

III. Continuous materials mechanical representation.

The paper of Nguyen Quoc Son will review typical examples of usual anelastic behaviours and will show that results recently achieved prove how successfull is already the approach which has just been outljned. Hut it remains that the mechanician is continously Eaced with the necessity to co more deeply inside the physics of the phenomena in order to find a realistic phenomenological description of the material involved in the structure he wants to study.

Let us mention a first answer with the use of rheological models for writing formally constitutive laws with some internal variables -which were often called "hidden variables" to underline that no direct physical significance was attached to them. For example, viscoelastic materials or perfectly elasto-plastic materials may be described as standard materials with a function $\varphi$ in (20) which is polynomial of degree 2 for the former and degree 1 sor the latter. The precise form of this function has to be obtained by "identification" of the results aiven by the model with experimental results. mhis process is sometimes still useful but cannot he considered to fulfil the requirements of a good mochanical representation. It is orecisely the purnose of this meeting to recoanize the basic mechanims of nlasticity with the hone that they may be taken into account in a reasonable wav in the constitutive laws. To build such continuous models rinich may be used by mechanicians for their studies $\Omega^{c}$ structures and which reflect in some respect the rroperties discovered by physicists or metalluraists is the main objective of what may be called the mechanic of continous materials. Let us close this introductory talk by some remarks.

III.1. Material invariance of perfectly elastic materials

The first one will stretch what can be called the material invariance of perfectlyelastic materials. As it is well known the thermodynamical potential -say the specific free energy for an isothermal evolution- is a convex (or more generally polyconvex) function. A regular problem for the equilibrium of a structure may be formulated as a variational problem which gives rise to a solution which may be looked as a function of the data -in general surface forces or displacements on the boundary. A global potential may be defined namely for the whole structure and the generalized Castigliano theorem tells that for a given structure in equilibrium, global potentials may be defined which may be derive from one of them by Legendre transforms (and consequently by couples of dual quantities) in the same way that specific thermodynamical potentials at each particle may be defined from one of them by Legendre transforms, the dual quantities at these levels 
being stress tensors and deformation tensors. This very well known property, which illustrates why classical thermodynamics may be formulated in a quite abstract and general way, has many important consequences. One of them is the possibility to schematize a highly non-homogeneous elastic body by another one with smoother properties by some process of homogeneization : the local properties at one "point" of the homogeneized body (macroscopic level) are the global properties of a "cell" which, at the microscopic level, has to be looked as an elastic continuum. It is the fundamental material invariance for perfectly elastic materials through the passage micro-macro.

III.2. Identifiable yeometrical or physical internal variables

The crucial difficulty in writing constitutive equations for anelastic materials is to choose the internal variables. In some cases, that can be done easily. It is possible, for instance, to write the global potential of an elastic structure with some well defined cracks : the geometrical shape appears as a (global) internal variable. In particular the dual quantity of the length of a rectilinear crack in a 2-dimensional elastic body is related to the stress concentration factor at the head of the cracl: and the propagation law of the crack may be given by a convenient pseudo-potential. The mechanics of the monocristal gives an example of a physical identifiable internal variable : the orthogonal tensor which gives the orientation of the crystallographic frame is conveniently introduced for the study of the elastoplastic monocrystal. This is the best example of what may be called quite generally a reactualized natural configuration, a concept which has been found quite useful in elastoplasticity at finite deformation where it is called the relaxed configuration. Even if such a choice of an internal variable is agreed, difficulties and controversial arguments may still arise when it must be decided how to compute time derivatives with respect to such a frame.

III.3. Micro-Macro mechanics

In general, one cannot hope to find a good representation of solid materials without taking account of the main physical phenomena. But solid materials are very often highly heterogeneous and, moreover, the mechanisms which rule the behaviour of the material are related to a very small scale. At the rresent time, jt is not nossible to take a rirect account on a very satisfactory way of these physical mechanisms identifiable at such a scale. Then, the microscale of the mechanician is still a macroscale for the solid state physicist. As a first consequence, one must not forget that the internal variables introduced in II.2 are in fact global variables for the microscopic description and more precisely those with a characteristic time of evolution comparable to the kinematic characteristic time of the particule - the others are either frozen or have their equilibrium value. As a second consequence, one must quite often be satisfied by some crude assumption at the microscopic level. In the localization - homogeneization scheme, one defines a reasonable model for the microcell and one derives the macroscopic behaviour with some further hypothesis, either on a statistical distribution or on a pseudoperiodicity property of the microcells. In the selfconsistent scheme, the mechanical interactions between a microcell and all adjacent ones are approximated by the interaction between this constituent and the equivalent homogeneous material whose behaviour is unknown. Performing this operation for each microcell, the unknown behaviour may be calculated by an averaging process.

Conclusion

This very short survey of some recent attempts or progress in the continuum mechanics of materials show how far we are still from a theory which will be able to incoporate the mechanisms of plasticity aiscovered by the solid state physicist into a continuum theory which will be able to answer all the questions of the mechanical engineer. If one is tempted to be discouraged by the size of the gap, one has to look back to the last two decades in order to note the very significant progress made by the continuum approach for facing the challenge that represents a full scientific understanding and representation of the behaviour of anelastic structiures. 